\title{
Accuracy of CT-Based Attenuation Correction in PET/CT Bone Imaging
}

\author{
Monica Abella1 ${ }^{1}$, Adam M. Alessio ${ }^{2}$, David A. Mankoff ${ }^{2}$, Lawrence R. MacDonald ${ }^{2}$, Juan \\ Jose Vaquero', Manuel Desco ${ }^{1,3,4}$, and Paul E. Kinahan ${ }^{2}$ \\ Monica Abella: mabella@mce.hggm.es \\ ${ }^{1}$ Dpto. de Bioingeniería e Ingeniería Aeroespacial. Universidad Carlos III de Madrid, Avda. de la \\ Universidad 30, 28911 Leganes, Madrid. Spain \\ ${ }^{2}$ Department of Radiology, University of Washington, 222 Fisheries Center (FIS), Box 357987, \\ Seattle, WA 98195-7987. USA \\ ${ }^{3}$ Unidad de Medicina Experimental. Hospital General Universitario Gregorio Marañón, Dr. \\ Esquerdo, 46. 28007, Madrid, Spain \\ ${ }^{4}$ Centro de Investigación en Red de Salud Mental (CIBERSAM, CIBER CB07/09/0031), 28007 \\ Madrid, Spain
}

\section{Abstract}

We evaluate the accuracy of scaling CT images for attenuation correction of PET data measured for bone. While the standard tri-linear approach has been well-tested for soft tissues, the impact of CT-based attenuation correction on the accuracy of tracer uptake in bone has not been reported in detail.

We measured the accuracy of attenuation coefficients of bovine femur segments and patient data using a tri-linear method applied to CT images obtained at different $\mathrm{kVp}$ settings. Attenuation values at $511 \mathrm{keV}$ obtained with a ${ }^{68} \mathrm{Ga} /{ }^{68} \mathrm{Ge}$ transmission scan were used as a reference standard. The impact of inaccurate attenuation images on PET standardized uptake values (SUVs) was then evaluated using simulated emission images and emission images from five patients with elevated levels of FDG uptake in bone at disease sites.

The CT-based linear attenuation images of the bovine femur segments underestimated the true values by $2.9 \pm 0.3 \%$ for cancellous bone regardless of $\mathrm{kVp}$. For compact bone the underestimation ranged from $1.3 \%$ at $140 \mathrm{kVp}$ to $14.1 \%$ at $80 \mathrm{kVp}$. In the patient scans at $140 \mathrm{kVp}$ the underestimation was approximately $2 \%$ averaged over all bony regions. The sensitivity analysis indicated that errors in PET SUVs in bone are approximately proportional to errors in the estimated attenuation coefficients for the same regions. The variability in SUV bias also increased approximately linearly with the error in linear attenuation coefficients.

These results suggest that bias in bone uptake SUVs of PET tracers range from 2.4\% to 5.9\% when using CT scans at 140 and $120 \mathrm{kVp}$ for attenuation correction. Lower $\mathrm{kVp}$ scans have the potential for considerably more error in dense bone. This bias is present in any PET tracer with bone uptake but may be clinically insignificant for many imaging tasks. However, errors from CTbased attenuation correction methods should be carefully evaluated if quantitation of tracer uptake in bone is important. 


\section{Introduction}

PET/CT has become an effective diagnostic tool in oncology imaging as it provides combined functional and anatomic imaging, resulting in improved lesion characterization and localization compared to PET alone (Beyer et al 2000; Wahl 2004).

An important synergy of PET/CT scanners is the use of the CT images for attenuation correction of the PET emission data (Kinahan et al 1998; Burger et al 2002; Kinahan et al 2003). There are several advantages of this approach compared to the earlier standard of PET transmission (TX) scans with ${ }^{68} \mathrm{Ga} /{ }^{68} \mathrm{Ge}$ sources. These include (i) a less noisy image, (ii) shorter acquisition times, and (iii) insensitivity of the attenuation image to emission contamination (Kinahan et al 2003). An important issue, addressed in this paper, is the potential bias due to the fact that CT data, acquired as a weighted average of photon energies ranging from approximately 30 to $140 \mathrm{KeV}$, have to be transformed into estimates of the attenuation coefficients of PET photon energies at $511 \mathrm{keV}$ (Burger et al 2002; Kinahan et al 2003; Ay et al 2011). Three main methods have been proposed to implement this conversion: dual-kVp CT scans, segmentation, and scaling. Dual-kVp (or dual-energy) CT scanning potentially allows for the most accurate approach (Kinahan et al 2006), but is complex and can increase patient radiation dose. Segmentation methods can also be complex, and have the potential to introduce bias (Schleyer et al 2010). The simplest and most commonly employed method is bi- or tri-linear scaling (Kinahan et al 1998; Burger et al 2002; Kinahan et al 2003), which closely approximates the electron density as a function of CT number in most tissues (Schneider et al 2000).

Quantitative PET images of tracer uptake in bone tissue are important for assessing both normal bone and cancer spread (Wahl et al 1991), as bone is a common site of metastasis (Stafford et al 2002). FDG PET/CT is commonly used for cancer staging, including the identification of bone metastases, and ${ }^{18} \mathrm{~F}$-fluoride PET/CT is increasingly used for bone imaging, including bone metastasis detection (Even-Sapir et al 2007). Accurate estimates of tracer uptake are particularly important for assessing bone metastases response to therapy, where FDG has shown considerable promise (Stafford et al 2002; Du et al 2007; Specht et al 2007; Meirelles et al 2010).

There are several studies presenting results on the accuracy of the linear attenuation coefficients derived from CT images in soft tissue, with or without contrast agents, in phantoms, human data, or small animals (e.g. (Burger et al 2002; Nakamoto et al 2002; Visvikis et al 2003; Berthelsen et al 2005; Mawlawi et al 2006)). However, the impact of CT-based attenuation correction (CTAC) on the accuracy of PET tracer uptake values for bony regions has not been carefully evaluated. A means of constructing a phantom that accurately mimics PET tracer uptake in both compact and cancellous bone has not yet been found. Cancellous bone is less dense but with a higher surface area than compact bone. It typically occupies the interior region of bones, is highly vascular, and frequently contains bone marrow.

The purpose of this work is to assess the errors from using the CT images for attenuation correction of PET data on estimates of tracer uptake in compact and cancellous bone. We performed three experiments using a combination of simulations, phantom studies, and patient data. Preliminary results were presented earlier (Abella et al 2007); here we refine the methods and extend the analysis of the results.

\section{Materials and Methods}

The bi-linear scaling method for PET/CT (Burger et al 2002; Kinahan et al 2003) assumes that all pixels in a CT image with CT numbers between approximately -1000 and $0 \mathrm{HU}$ are 
a mixture of air and water, while pixels with a CT number greater than $0 \mathrm{HU}$ are composed of a mixture of water and bone. The theory is described in detail elsewhere (Kinahan et al 2003). Our experiments were based on the Discovery STE PET/CT scanner (GE Healthcare, Waukesha, WI), which uses a tri-linear scaling approach. With this method the linear attenuation coefficient at $511 \mathrm{keV}$ (LAC) is estimated by $L A C=a \times C T+b$, where $a$ and $b$ are constants listed in Table 1 (Conversion factors were determined from internal scanner calibration factors and were derived based on the work by Lonn 2003) and "CT" is the CT number in Hounsfield units (HU). The corresponding conversions are illustrated in Figure 1.

Images were reconstructed with filtered backprojection (FBP) using a $5 \mathrm{~mm}$ Hanning window. All simulations and image based analysis were performed with IDL version 6.4 (ITT Visual Information Solutions, Boulder, CO).

The primary measure of tracer uptake used was the standardized uptake value (SUV): $S U V$ $=R /\left(D^{\prime} / \tilde{V}\right)$, where $R(\mathrm{kBq} / \mathrm{ml})$ is the activity concentration estimated from the region of interest (ROI), $D^{\prime}(\mathrm{kBq})$ is the decay-corrected injected dose, and $\widetilde{V}$ is a surrogate for the volume of distribution of tracer inside the body, in our case body weight $(\mathrm{g})$. In this case the SUV units were $\mathrm{g} / \mathrm{ml}$.

\subsection{Experiment 1: CT-derived attenuation bias}

The goal of the first experiment was to determine the bias in the CT-derived attenuation coefficients of bone in large portions, immune to partial volume errors (bovine phantom) and in realistic human-sized bone regions (limited patient study). To this end, we compared the linear attenuation coefficients derived from the CT image (LAC $\mathrm{CTAC}_{\mathrm{C}}$ ) by means of the tri-linear transform (Figure 1) with those obtained from a 2D PET transmission image $\left(\mathrm{LAC}_{\mathrm{TX}}\right)$ using a ${ }^{68} \mathrm{Ga} /{ }^{68} \mathrm{Ge}$ positron source.

We performed a single scan of three frozen bovine femur segments positioned in a $20 \times 20$ $\mathrm{cm}$ cylindrical phantom. The phantom also contained a $5 \mathrm{~cm}$ diameter cylinder with dilute iodine-based contrast agent as a reference. A 10 min 2D PET transmission scan was acquired on a GE Advance PET Scanner (GE Healthcare, Waukesha, WI) and reconstructed with the FBP algorithm with a $5 \mathrm{~mm}$ Hanning window, obtaining a set of sixty five $4.25 \mathrm{~mm}$ thick slices, each $128 \times 128$ pixels of size $2.3 \mathrm{~mm} \times 2.3 \mathrm{~mm}$. A CT scan of the same phantom was acquired with the GE Discovery STE PET/CT scanner using $140 \mathrm{kVp}, 120 \mathrm{kVp}$, and 80 $\mathrm{kVp}$ with $200 \mathrm{mAs}$. The attenuation coefficients from CT were calculated from a filtered version of the CT image covering a FOV of $70 \mathrm{~cm}$, yielding a data set of $73 \times 3.75 \mathrm{~mm}$ image slices, each with $512 \times 512$ pixels of size $1.37 \mathrm{~mm} \times 1.37 \mathrm{~mm}$. The CT image was then downsampled (with linear interpolation) to match the PET transmission pixel size and both images were aligned manually as shown in Figure 2. This final phase of the CT image converted to a PET attenuation map is termed the CTAC image. The accuracy of alignment was visually assessed to be better than $2 \mathrm{~mm}$ in all directions. Both the CTAC and TX images represent the spatial distribution of the linear attenuation coefficients at $511 \mathrm{keV}$ with units of $\mathrm{cm}^{-1}$.

Five volumetric regions of the bones were segmented by thresholding and erosion of the CT image to get equivalent volume masks for the PET TX and CTAC images. Two regions were from compact bone, two were from cancellous bone, and one was from the contrast cylinder. Two of these volume masks are shown in Supplemental Figure 1 (supplemental materials are available online only). Differences between the average linear attenuation coefficients within each region from the two images were then calculated. The numerical results are reported as the percent difference in the average linear attenuation coefficients at $511 \mathrm{keV}$ by: 


$$
\Delta_{L A C}=\frac{100 \% \times\left(L A C_{C T A C}-L A C_{T X}\right)}{L A C_{T X}}
$$

We repeated the above analysis for previously-acquired scans of a patient from both scanners using $140 \mathrm{kVp}$ for the CT scan component. The PET transmission image was acquired pre-injection. All patient data was analyzed under Institutional Review Board (IRB) approval for retrospective patient data analysis. Figure 3 shows the CTAC and TX images of this patient.

In this case, it was not possible to accurately register both images using rigid transformations and use the CT-derived region mask. Instead, we calculated the mean linear attenuation coefficient for the entire bone region in both data sets using thresholding. It was determined experimentally that thresholds of $0.1070 \mathrm{~cm}^{-1}$ for CTAC and $0.1074 \mathrm{~cm}^{-1}$ for PET TX images obtained very similar regions (volumes of 87.7 and $87.2 \mathrm{~cm}^{3}$ ). In order to assess the consistency of results using this method, we repeated the same process with the bovine femur phantom image regions to compare with the analysis using the CT-derived region mask.

\subsection{Experiment 2: Evaluation of the bias in simulated PET tracer uptake due to inaccuracies in measured CTAC images}

In the second experiment, we evaluated the impact of inaccuracies in the CTAC image on the estimated tracer SUV in the corresponding PET image. Due to the difficulty of introducing a known quantity of FDG into the bovine bone, we instead simulated an emission image by thresholding the CT image to determine bone regions and then assigned different activity values to each segmented region (Figure 4). The assigned PET tracer uptake values are given in Table 2 . To simplify the analysis we removed the contrast cylinder and the smaller bone (\#3) from the bovine phantom images.

The effect of incorrect CTAC factors was determined by forward-projecting the simulated PET image, including attenuation (by using the inverse of the 'true' attenuation correction factors obtained from the PET transmission scan), and correcting for attenuation with the 'erroneous' attenuation correction factors (obtained from the CTAC image). The attenuation corrected data were then reconstructed with FBP. The process is outlined in Figure 5.

For a reference image, we forward-projected and reconstructed the simulated emission data. In this manner the sampling and resolution effects were the same for the two PET emission images. The numerical results calculated were the percent difference in the average SUV within each region between the PET emission images using the PET TX scan ( $\left.\mathrm{SUV}_{\mathrm{TX}}\right)$ and CTAC scan ( $\left.\mathrm{SUV}_{\mathrm{CTAC}}\right)$ attenuation correction factors as follows:

$$
\Delta_{S U V}=\frac{100 \% \times\left(S U V_{C T A C}-S U V_{T X}\right)}{S U V_{T X}}
$$

\subsection{Experiment 3: Sensitivity analysis using patient emission images}

In the third experiment, we used CT and PET images from five patient exams with significant FDG uptake in bone tissue. Similar to Experiment 2 we measured the bias of the FDG uptake due to simulated errors in attenuation correction. The experimental process is outlined in Figure 6. Since patients were imaged only on the PET/CT scanner (Figure 7 shows one of these studies), $511 \mathrm{keV}$ PET TX images were not available. For these patient exams we used the measured $\mathrm{LAC}_{\mathrm{CTAC}}$ and SUV images as the reference standards. 
The values used for comparison in each selected site corresponding to a bone metastasis were expressed in terms of maximum SUV (SUV MAX) and mean value $\left(\mathrm{SUV}_{75}\right)$ in a contiguous volume of interest (VOI) covering all voxels with SUVs at least $75 \%$ of SUV $_{\text {MAX }}{ }^{1}$. We used twelve VOIs selected from the five studies of pre-treatment breast cancer patients with extensive bone metastases in the thoracic spine, lumbar spine, and pelvis.

The VOIs were obtained by the following procedure. First a bone mask image was segmented by thresholding the CT image after resampling and filtering to match the PET image resolution. Cylindrical VOIs were then manually defined to select specific bony areas with elevated FDG uptake. The tumor volume values were then obtained by including the voxels in the intersection of both the cylindrical VOIs and the bone image mask with values higher than $75 \%$ of SUV MAX (Figure 7). We measured the dependence of the error in SUV $_{\text {CTAC images as a function of the estimated SUV }}$ MAX values, as well as the dependence on introduced errors in $\mathrm{LAC}_{\mathrm{CTAC}}$ (we tested errors in $\mathrm{LAC}_{\mathrm{CTAC}}$ of 2, 4, 6 and 8\%).

\section{Results}

\subsection{Experiment 1: CT-derived attenuation bias}

Table 3 shows the error in linear attenuation coefficients (Equation 1) measured for the five volumes segmented in the bovine femur phantom. In this case the values from the PET transmission scan are used as reference standard.

Errors in linear attenuation coefficients $\left(\Delta_{\mathrm{LAC}}\right)$ for cancellous bone were $2.9 \pm 0.3 \%$ regardless of the $\mathrm{kVp}$ used during $\mathrm{CT}$ acquisition. For compact bone the underestimation ranged from approximately $1.4 \%$ at $140 \mathrm{kVp}$ to $13.8 \%$ at $80 \mathrm{kVp}$. The alternative region selection method yielded $\Delta_{\mathrm{LAC}}$ of $-2.0 \pm 0.2 \%$ in both the patient 1 scan and the bovine phantom at $140 \mathrm{kVp}$, confirming consistency between the phantom and patient results. The errors for the contrast cylinder were significantly higher at $27.2 \%$.

\subsection{Experiment 2: Evaluation of the bias in simulated PET tracer uptake due to inaccuracies in measured CTAC images}

The differences between the assigned SUV (Table 2) and SUV $\mathrm{SX}_{\mathrm{TX}}$ in Table 4 reflect the effects of the numerical processing. For this reason the SUVs calculated using CT based attenuation correction ( $\mathrm{SUV}_{\mathrm{CTAC}}$ ) are compared to $\mathrm{SUV}_{\mathrm{TX}}$ as described in Equation 2. As indicated in Table 4, the underestimation of PET SUV when using biased LAC $_{\mathrm{CTAC}}$ values (using the PET transmission image $\mathrm{LAC}_{\mathrm{TX}}$ as a gold standard) ranged from $-2.4 \%$ to $-5.9 \%$ at $140 \mathrm{kVp}$ and $120 \mathrm{kVp}$.

\subsection{Experiment 3: Sensitivity analysis using patient images}

The estimated bias in $\mathrm{SUV}_{\text {MAX }}$ as a function of 'true' $\mathrm{SUV}_{\mathrm{MAX}}$ is presented in Figure $8 \mathrm{~A}$ for each $\mathrm{LAC}_{\mathrm{CTAC}}$ bias $(-2 \%,-4 \%,-6 \%$, and $-8 \%)$ introduced to the twelve lesions from five patient emission images. Here 'true' $\mathrm{SUV}_{\mathrm{MAX}}$ was found using the default $\mathrm{LAC}_{\mathrm{CTAC}}$ because $\mathrm{LAC}_{\mathrm{TX}}$ were not available for these images.

When the results are averaged for all SUV values (Figure 8B) there is an approximate error in $\mathrm{SUV}_{\mathrm{MAX}}$ of $1 \%$ for each percentage error in $\mathrm{LAC}_{\mathrm{CTAC}}$, which is in agreement with our previously reported results for soft tissue (Kinahan et al 2006). The variability in the SUV bias also increased approximately linearly with the error in $\mathrm{LAC}_{\mathrm{CTAC}}$. (Figure $8 \mathrm{~B}$ ). There

\footnotetext{
${ }^{1}$ The subscripts SUVTX and SUVCTAC are used to differentiate between the attenuation correction methods used in generating SUVs, while SUVMAX and SUV75 indicate the method of measuring SUVs.
} 
was a weak inverse dependence of bias in SUV MAX on the true SUV value (Figure 8A).

Similar results to those described above for $\mathrm{SUV}_{\mathrm{MAX}}$ were found for $\mathrm{SUV}_{75}$.

\section{Discussion}

Photon attenuation and its correction are non-linear processes (Bai et al 2003), and thus it is challenging to characterize errors in attenuation correction. The use of CT-based attenuation correction adds an additional level of complexity due to the inability of CT to provide accurate measures of attenuation (Hsieh 2009). There are several factors such as tumor size and tumor-background ratio that may alter how errors in attenuation correction affect PET image quantitation in bone. Our initial evaluations, which avoided partial volume effects, indicated that PET image biases are linearly dependent on errors in CT-based estimates of bone attenuation coefficients when those errors are $<10 \%$, and also slightly dependent on the magnitude of true SUV. We therefore focused on the impact of these effects.

There are significant technical challenges in measuring quantitative errors in PET tracer uptake in bone when CT-based attenuation correction is used. Appropriate phantom design is difficult (e.g. tracer uptake in bony structures), and patient-based comparisons are confounded by changes in tracer uptake duration, non-rigid image registration, and the typically resolution-limited measurements. To explicitly evaluate the mechanisms that introduce error into estimated tracer uptake values in bone when CT-based attenuation correction is used, we performed three experiments using simulations, phantoms, and clinical data. Experiment 1 determined the bias in the CT derived attenuation images. Experiment 2 determined the bias in simulated PET images due to measured errors in attenuation correction, not confounded by partial volume effects. Experiment 3 determined the bias in PET images due to errors in attenuation correction for realistic attenuation and emission distributions.

We first determined the estimated linear attenuation coefficients $\left(\mathrm{LAC}_{\mathrm{CTAC}}\right)$ at different $\mathrm{kVp}$ (Table 3). For cancellous bone the errors in linear attenuation coefficients $\left(\Delta_{\mathrm{LAC}}\right)$ were approximately $-3 \%$ regardless of the $\mathrm{kVp}$ used using $\mathrm{CT}$ acquisition. For compact bone the lowest error in $\mathrm{LAC}_{\mathrm{CTAC}}$ of $-1.4 \%$ was obtained at $140 \mathrm{kVp}$. As the $\mathrm{kVp}$ decreased to 80 $\mathrm{kVp}, \Delta_{\mathrm{LAC}}$ increased to approximately $-14 \%$. At $120 \mathrm{kVp}$, arguably the most common voltage used in CT, $\Delta_{\text {LAC }}$ was approximately $-7 \%$ in compact bone. The standard deviation in the average LAC value was calculated, as opposed to a root mean square (RMS) error, as we are concerned about the bias effects in the CTAC values, as opposed to the noise in the PET TX values. We used the manufacturer's trilinear slope and intercept parameters (table 1) to evaluate error for an actual system. Considering that all estimated $\mathrm{LAC}_{\mathrm{CTAC}}$ values were below the reference standard for compact bone, the conversion parameters (slope) could be increased for CT numbers above $1000 \mathrm{HU}$ for all regions to reduce bias. Results suggest that the conversion factors for the lower $\mathrm{kVp}$ acquisitions may need to be increased more than the higher $\mathrm{kVp}$ studies. It will be more challenging to improve the conversion for cancellous bone considering it has CT numbers in the range of water (-100 to $100 \mathrm{HU})$. Adjustments to the conversion for this range may lead to increased errors in non-bone, softtissue regions. Evaluation of the combined bone regions revealed an error of $-2 \%$ for both the bovine femur phantom and the patient image at $140 \mathrm{kVp}$. This error is between the errors for compact and cancellous bone at $140 \mathrm{kVp}$. Thus the error in $\mathrm{LAC}_{\mathrm{CTAC}}$ at $120 \mathrm{kVp}$ can be expected to vary between $-3 \%$ and $-7 \%$, although this will vary based on the mixture of bone type and other factors (e.g. object size/shape and partial volume effects).

As expected, values for the region with CT contrast agent showed the largest error, since the conversion curves applied are intended for images without contrast. The application of trilinear CTAC conversion curves specific for contrast media (Lonn 2003) lowered these 
errors in contrast areas at the expense of dramatically increasing the error in bone areas (data not shown). Therefore, for studies with contrast media and where bone uptake is expected, a different approach, such as a dual energy method or hybrid classification/scaling algorithm, may be advisable for converting the CT values to LAC values at PET energies (Kinahan et al 2006). The effects of CT contrast agents were not considered any further in this study.

Using the expected range of errors in $\mathrm{LAC}_{\mathrm{CTAC}}$ for bone, we then evaluated the effect on estimated SUV values of the bovine femur phantom (Table 4). The differences between the assigned SUV in Table 2 and SUV $_{\mathrm{TX}}$ in Table 4 reflect the effects of the numerical processing (forward projection, attenuation, and image reconstruction). For this reason the SUVs calculated using CT based attenuation correction ( $\mathrm{SUV}_{\mathrm{CTAC}}$ ) are compared to $\mathrm{SUV}_{\mathrm{TX}}$ (Equation 2 and Figures 5 and 6) so that both estimates have the same effects from numerical processing. At $140 \mathrm{kVp}$, the estimated $\mathrm{SUV}_{\mathrm{CTAC}}$ was lower than the reference $\mathrm{SUV}_{\mathrm{TX}}$ value by approximately $2.5 \%$ in compact bone and 2.4 to $4.1 \%$ in cancellous bone.

While the results from Tables 3 and 4 determine the impact of errors in $\mathrm{LAC}_{\mathrm{CTAC}}$ on PET SUVs, they do not take into account the important effects of the location and size of attenuating material in patient images. To include these effects and perform a more extended sensitivity analysis we used five additional patient images with significant FDG uptake in bone lesions (e.g. Figure 7). This allowed estimation of the error in FDG uptake as a function of the error in the CT-based attenuation correction and nominal SUV uptake as shown in Figure 8A. From Figure 8B we see that the percent error in PET SUV MAX is roughly proportional to the percent error in $\mathrm{LAC}_{\mathrm{CTAC}}$. Very similar results were found for SUV $_{75}$ (data not shown). These results are consistent with our previous results for soft tissues (Kinahan et al 2006). As expected, the variability of SUV measurements increased with increasing error in LAC factors because of the propagation of error from multiplicative terms.

Based on Figure $8 \mathrm{~B}$, with a typical error in $\mathrm{LAC}_{\mathrm{CTAC}}$ of up to $-3.1 \%$ at $140 \mathrm{kVp}$ and up to $-6.8 \%$ at $120 \mathrm{kVp}$, we would thus expect an $\mathrm{SUV}_{\mathrm{MAX}}$ error of approximately $-3.1 \%$ and $-6.8 \%$ at 140 and $120 \mathrm{kVp}$. This represents a range for PET/CT imaging parameters on clinical scanners using the tri-linear transform method described above (Figure 1 and Table $1)$.

Very similar results were found for SUV MAX and SUV 75 . The definition of the $\mathrm{SUV}_{75}$ metric was motivated by several factors. It is generally not feasible to define a mean SUV for bone lesions since the boundaries are difficult to determine by either PET or anatomic imaging such as CT. Likewise, our goal was not to compensate for resolution loss along boundaries of lesions (Soret et al 2007), which can lead to substantial variability in different definitions of mean SUV, but rather to assess the underlying bias from attenuation correction. This $\mathrm{SUV}_{75}$ metric provides a small amount of local averaging to reduce the

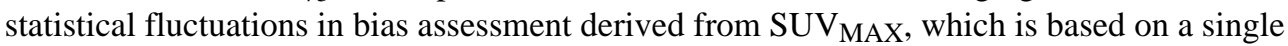
voxel. Other approaches, such as a small fixed size ROI, e.g. $\mathrm{SUV}_{\text {peak }}$, may also be useful in this regard (Velasquez et al 2009; Wahl et al 2009).

To compare our results with those previously published, we note that, as far as we know, no study reports both the errors in attenuation coefficients and their propagation to PET tracer uptake in bone. Burger et al. (Burger et al 2002) demonstrated an overall qualitative agreement in estimated linear attenuation coefficient at $511 \mathrm{keV}$ (LAC $\mathrm{CTAC}_{\text {CT }}$ ) using the bilinear method for 114 mixed soft tissue and bone regions in patient studies, but there was no differentiation of bone versus soft tissue results for emission data. Inspection of the key results of the Burger study show little significant bias for $\mathrm{LAC}_{\mathrm{CTAC}}$ versus $\mathrm{LAC}_{\mathrm{TX}}$ for a range of CT values that should encompass bone, i.e. in the range of 200 to $800 \mathrm{HU}$, although 
there is some variability in the results. In addition, a single measurement with a bovine femur segment showed a slight bias of $\mathrm{LAC}_{\mathrm{CTAC}}$ versus $\mathrm{LAC}_{\mathrm{TX}}$. Thus the results of the Burger study appear to be consistent with those presented here. In contrast, Visvikis et al. (Visvikis et al 2003) found that with a similar implementation of the bi-linear scaling method, CT-based attenuation coefficients $\left(\mathrm{LAC}_{\mathrm{CTAC}}\right)$ were higher by $4.5 \%$ than those measured using a ${ }^{68} \mathrm{Ga} /{ }^{68} \mathrm{Ge}$ transmission source for cortical bone. This study analyzed the impact on PET tracer uptake values for lung and soft tissue, but not in bone regions.

The study by Nakamoto el at. (Nakamoto et al 2002) evaluated the impact of CTAC versus ${ }^{68} \mathrm{Ga} /{ }^{68} \mathrm{Ge}$ transmission sources on measured PET tracer values, but did not report on differences in LAC. They found that calculated radioactivity concentrations were significantly greater in osseous lesions than in non-osseous lesions $(11.0 \%$ vs. $2.3 \%$ for mean value in an ROI, based on $n=9$ bone lesions). A weak positive correlation was observed between the CT Hounsfield units within the regions of interest and the percentage difference in apparent tracer activity in the CT-corrected images. They hypothesized that errors exist in the conversion of high CT values to $511-\mathrm{keV}$ attenuation coefficients. As we have shown here, such errors would impact estimation of PET tracer uptake in bone. The errors reported by Nakamoto are higher than those reported on our study. The reason for this difference is not clear, in particular as similar PET/CT scanners were used, but it could be due to the small sample size, differences in the tri-linear scaling parameters, calibration of the CT scanner, or differences in the processing of the CTAC images. In addition, a segmented attenuation correction method was used to suppress noise from the 3 minute PET transmission scan, which can render low density bone as soft tissue. Furthermore, we reconstructed PET images with filtered-backprojection (FBP), whereas the study by Nakamoto el at. used ordered-subsets expectation-maximization (OSEM). Due to the nonlinear nature of OSEM, its behavior when there are errors in data is unpredictable. Nakamoto et al. suggest using filtered backprojection based germanium-corrected images as a standard for a more direct error assessment (Visvikis et al 2003). However, since FBP and OSEM provide accurate results when attenuation (and other) corrections are applied, we expect in general that quantitative results for FBP and OSEM to be similar in the presence of CTAC bias.

For diagnostic purposes the small errors in estimated PET tracer values will most likely not influence clinical interpretation (Du et al 2007; Tateishi et al 2008). The underestimation of the attenuation correction factors should lead to fixed biases in the SUVs, and with a variability that is below reported reproducibility for SUV measurements for PET imaging (Boellaard 2009). However, if PET/CT imaging is being used to evaluate response to therapy, then the bias in the estimation of PET tracer uptake may well become significant. Since all positron emitting tracers have the same annihilation energy of $511 \mathrm{keV}$, these results will apply to all PET tracers, including ${ }^{18} \mathrm{~F}-\mathrm{NaF}$ for bone lesion imaging (Even-Sapir et al 2007).

\section{Conclusion}

Our results show that the percent error in PET SUV for bone imaging is roughly proportional to the percent error in the linear attenuation coefficients estimated from the CT scan $\left(\mathrm{LAC}_{\mathrm{CTAC}}\right)$. The errors in bone $\mathrm{LAC}_{\mathrm{CTAC}}$ in turn, were in the range of $-1.3 \%$ to $-6.8 \%$ at 140 and $120 \mathrm{kVp}$, depending on the experiment and type of analysis performed. Thus we expect an error of $-1.3 \%$ to $-6.8 \%$ for PET tracer uptake values in bone lesions over a wide range of SUVs, with smaller errors for the higher $\mathrm{kVp}$ scans. Errors increased for cortical bone as the scan $\mathrm{kVp}$ was reduced. While errors in estimated PET tracer values most likely do not influence clinical interpretation, quantitative studies evaluating therapeutic response in bone should account for changes in bone composition. These results 
will apply to all PET tracers and are based on the specific tri-linear scaling method implemented in the PET/CT scanner we used for the experimental studies (Figure 1, Table 1). On other PET/CT scanners there are variations in how the scaling method is implemented. For those systems these results should be re-evaluated.

\section{Supplementary Material}

Refer to Web version on PubMed Central for supplementary material.

\section{Acknowledgments}

We gratefully acknowledge the help of the meat department staff at the Sand Point Metropolitan Market (Seattle, WA) for the preparation of the bovine femur phantom. We also acknowledge helpful discussions with Steven Kohlmyer of GE Healthcare Technologies. This work was supported by grants EC2004-07052-C02 (Ministerio de Educación y Ciencia), CD-TEAM (Ministerio de Industria) and GR/SAL/024104 (Comunidad de Madrid), and NIH grants R01-CA115870 and R01CA124573.

\section{References}

Abella M, Mankof D, Vaquero JJ, Desco M, Kinahan PE. Accuracy of CT-based attenuation correction in bone imaging with PET/CT. IEEE Nucl Sci Symp Conf Rec. 2007; 6:4485-4488.

Ay RM, Shirmohammad M, Sarkar S, Rahmim A, Zaidi H. Comparative Assessment of EnergyMapping Approaches in CT-Based Attenuation Correction for PET. Mol Ima Biol. 2011; 13:187198.

Bai C, Kinahan PE, Brasse D, Comtat C, Townsend DW, Meltzer CC, Villemagne V, Charron M, Defrise M. An Analytic Study of the Effects of Attenuation on Tumor Detection in Whole-Body PET Oncology Imaging. J Nucl Med. 2003; 44(11):1855-1861. [PubMed: 14602870]

Berthelsen AK, Holm S, Loft A, Klausen TL, Andersen F, Højgaard L. PET/CT with intravenous contrast can be used for PET attenuation correction in cancer patients. Eur J Nucl Med Mol Imaging. 2005; 32(10):1167-1175. [PubMed: 15909196]

Beyer T, Townsend D, Brun T, Kinahan P, Charron M, Roddy R, Israel J, Jerin J, Young J, Byars L, Nutt R. A combined PET/CT scanner for clinical oncology. J Nucl Med. 2000; 41:1369-1379. [PubMed: 10945530]

Boellaard R. Standards for PET Image Acquisition and Quantitative Data Analysis. J Nucl Med. 2009; 50:11S-20S. [PubMed: 19380405]

Burger C, Goerres G, Schoenes S, Buck A, Lonn AH, Von Schulthess GK. PET attenuation coefficients from CT images: experimental evaluation of the transformation of CT into PET 511keV attenuation coefficients. Eur J Nucl Med Mol Imaging. 2002; 29(7):922-927. [PubMed: 12111133]

Du Y, Cullum I, Illidge TM, Ell PJ. Fusion of Metabolic Function and Morphology: Sequential [18F]Fluorodeoxyglucose Positron-Emission Tomography/Computed Tomography Studies Yield New Insights Into the Natural History of Bone Metastases in Breast Cancer. J Clinical Oncology. 2007; 25(23):3440-3447.

Even-Sapir E, Mishani E, Flusser G, Metser U. 18F-Fluoride Positron Emission Tomography and Positron Emission Tomography/Computed Tomography. Semin Nucl Med. 2007; 37(6):462-469. [PubMed: 17920353]

Hsieh, J. Computed Tomography: Principles, Design, Artifacts, and Recent Advances, Second Edition (SPIE Press Monograph Vol PM188). SPIE Publications; 2009.

Kinahan P, Alessio A, Fessler J. Dual Energy CT Attenuation Correction Methods for Quantitative Assessment of Response to Cancer Therapy with PET/CT Imaging. Technol Cancer Res Treat. 2006; 5(4):319-328. [PubMed: 16866562]

Kinahan P, Hasegawa B, Beyer T. X-ray-based attenuation correction for positron emission tomography/computed tomography scanners. Semin Nucl Med. 2003; 33(3):166-179. [PubMed: 12931319] 
Kinahan PE, Townsend DW, Beyer T, Sashin D. Attenuation correction for a combined 3D PET/CT scanner. Med Phys. 1998; 25(10):2046-2053. [PubMed: 9800714]

Lonn AHR. Evaluation of method to minimize the effect of X-ray contrast in PET-CT attenuation correction. IEEE Nucl Sci Symp Conf Rec. 2003; 3(19):2220-2221.

Mawlawi O, Erasmus JJ, Munden RF, Pan T, Knight AE, Macapinlac HA, Podoloff DA, Chasen M. Quantifying the Effect of IV Contrast Media on Integrated PET/CT: Clinical Evaluation. Am J Roentgenol. 2006; 186(2):308-319. [PubMed: 16423932]

Meirelles GS, Schöder H, Ravizzini GC, Gönen M, Fox JJ, Humm J, Morris MJ, Scher HI, Larson SM. Prognostic value of baseline [18F] fluorodeoxyglucose positron emission tomography and 99mTc-MDP bone scan in progressing metastatic prostate cancer. Clin Cancer Res. 2010; 16(24): 6093-6099. [PubMed: 20975102]

Nakamoto Y, Osman M, Cohade C, Marshall LT, Links JM, Kohlmyer S, LWR. PET/CT: Comparison of Quantitative Tracer Uptake Between Germanium and CT Transmission Attenuation-Corrected Images. J Nucl Med. 2002; 43(9):1137-1143. [PubMed: 12215550]

Schleyer PJ, Schaeffter T, KMP. The effect of inaccurate bone attenuation coefficient and segmentation on reconstructed PET images. Nucl Med Commun. 2010; 31(8):708-716. [PubMed: 20505553]

Schneider W, Bortfeld T, Schlegel W. Correlation between CT numbers and tissue parameters needed for Monte Carlo simulations of clinical dose distributions. Phys Med Biol. 2000; 45:459-478. [PubMed: 10701515]

Soret M, Bacharach SL, Buvat I. Partial-Volume Effect in PET Tumor Imaging. J Nucl Med. 2007; 48(6):932-945. [PubMed: 17504879]

Specht J, Tam S, Kurland B, Gralow J, Livingston R, Linden H, Ellis G, Schubert E, Dunnwald L, Mankoff D. Serial 2-[18F] fluoro-2-deoxy-D-glucose positron emission tomography (FDG-PET) to monitor treatment of bone-dominant metastatic breast cancer predicts time to progression (TTP). Breast Cancer Res Treat. 2007; 105(1):87-94. [PubMed: 17268819]

Stafford S, Gralow J, Schubert E, Rinn K, Dunnwald L, Livingston R, Mankoff D. Use of serial FDG PET to measure the response of bone-dominant breast cancer to therapy. Acad Radiol. 2002; 9(8): 913-921. [PubMed: 12186440]

Tateishi U, Gamez C, Dawood S, Yeung HWD, Cristofanilli M, Macapinlac HA. Bone Metastases in Patients with Metastatic Breast Cancer: Morphologic and Metabolic Monitoring of Response to Systemic Therapy with Integrated PET/CT. Radiology. 2008; 247(1):189-196. [PubMed: 18372468]

Velasquez LM, Boellaard R, Kollia G, Hayes W, Hoekstra OS, Lammertsma AA, Galbraith SM. Repeatability of 18F-FDG PET in a Multicenter Phase I Study of Patients with Advanced Gastrointestinal Malignancies. J Nucl Med. 2009; 50:1646-1654. [PubMed: 19759105]

Visvikis D, Costa DC, Croasdale I, Lonn AHR, Bomanji J, Gacinovic S, Ell PJ. CT-based attenuation correction in the calculation of semi-quantitative indices of [18F]FDG uptake in PET. European Journal of Nuclear Medicine and Molecular Imaging. Eur J Nucl Med and Mol Ima. 2003; 30(3): 344-353.

Wahl R. Why nearly all PET of abdominal and pelvic cancers will be performed as PET/CT. J Nucl Med. 2004; 45:82S-95S. [PubMed: 14736839]

Wahl RL, Cody RL, Hutchins GD, Mudgett EE. Primary and metastatic breast carcinoma: initial clinical evaluation with PET with the radiolabeled glucose analogue 2-[F-18]-fl uoro-2-deoxy-Dglucose. Radiology. 1991; 179:765-770. [PubMed: 2027989]

Wahl RL, Jacene H, Kasamon Y, Lodge MA. From RECIST to PERCIST: Evolving Considerations for PET response criteria in solid tumors. J Nucl Med. 2009; 50(1):122S-150S. [PubMed: 19403881] 


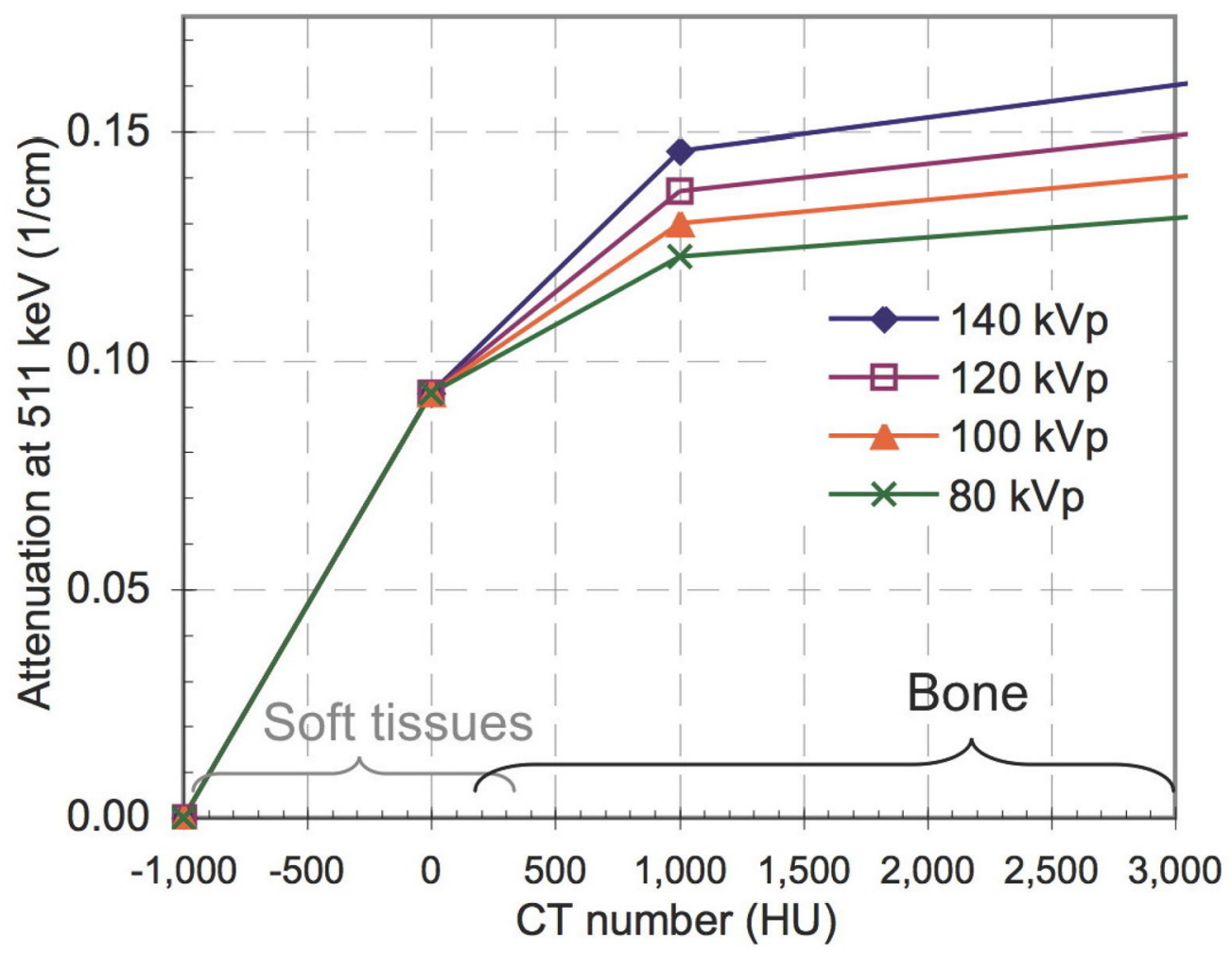

Figure 1.

Tri-linear scaling method used to convert CT numbers to linear attenuation coefficients at $511 \mathrm{keV}$ for different $\mathrm{x}$-ray tube potentials (values provided by the manufacturer).

Approximate ranges for CT numbers of soft tissues and osseous tissues are indicated. 


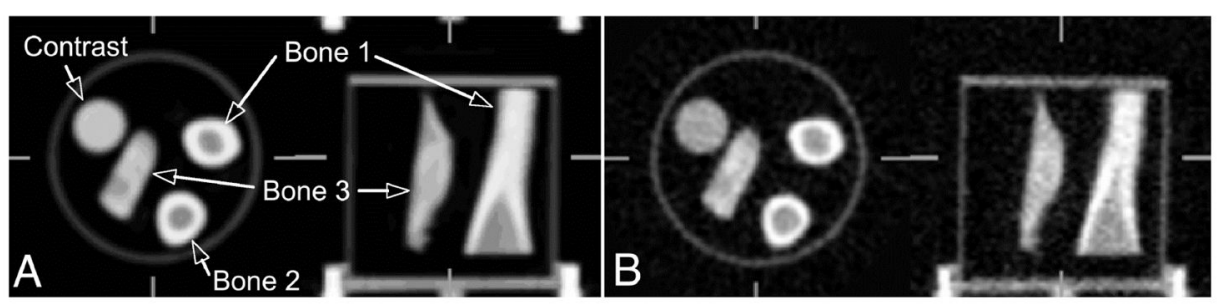

Figure 2.

Axial and sagittal views of frozen bovine femur segments in a $20 \times 20 \mathrm{~cm}$ cylindrical phantom. A: CT-based attenuation correction (CTAC) image $(140 \mathrm{kVp})$ interpolated to match the PET transmission resolution and after scaling attenuation coefficients to $511 \mathrm{keV}$ using the tri-linear transform (Figure 1, Table 1). B: 10 min PET transmission (TX) image at $511 \mathrm{keV}$ used as a reference standard. 


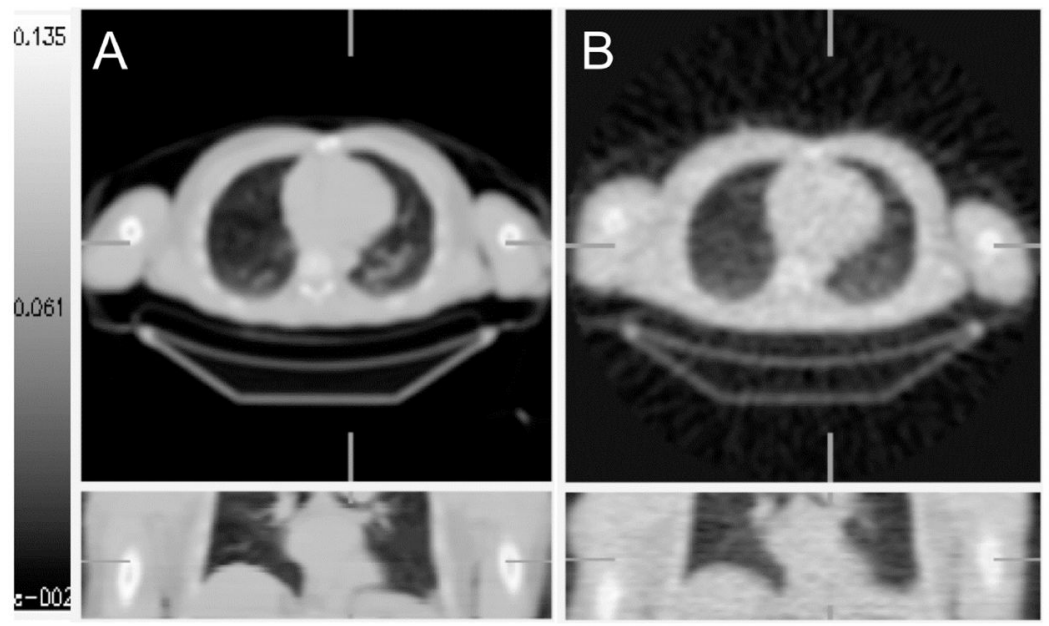

Figure 3.

Axial and coronal views of the CTAC (A) and PET TX (B) images of patient 1 scan. Grey scale units are for the linear attenuation coefficient at $511 \mathrm{keV}$ with units of $1 / \mathrm{cm}$. 


\section{Bovine femur phantom}
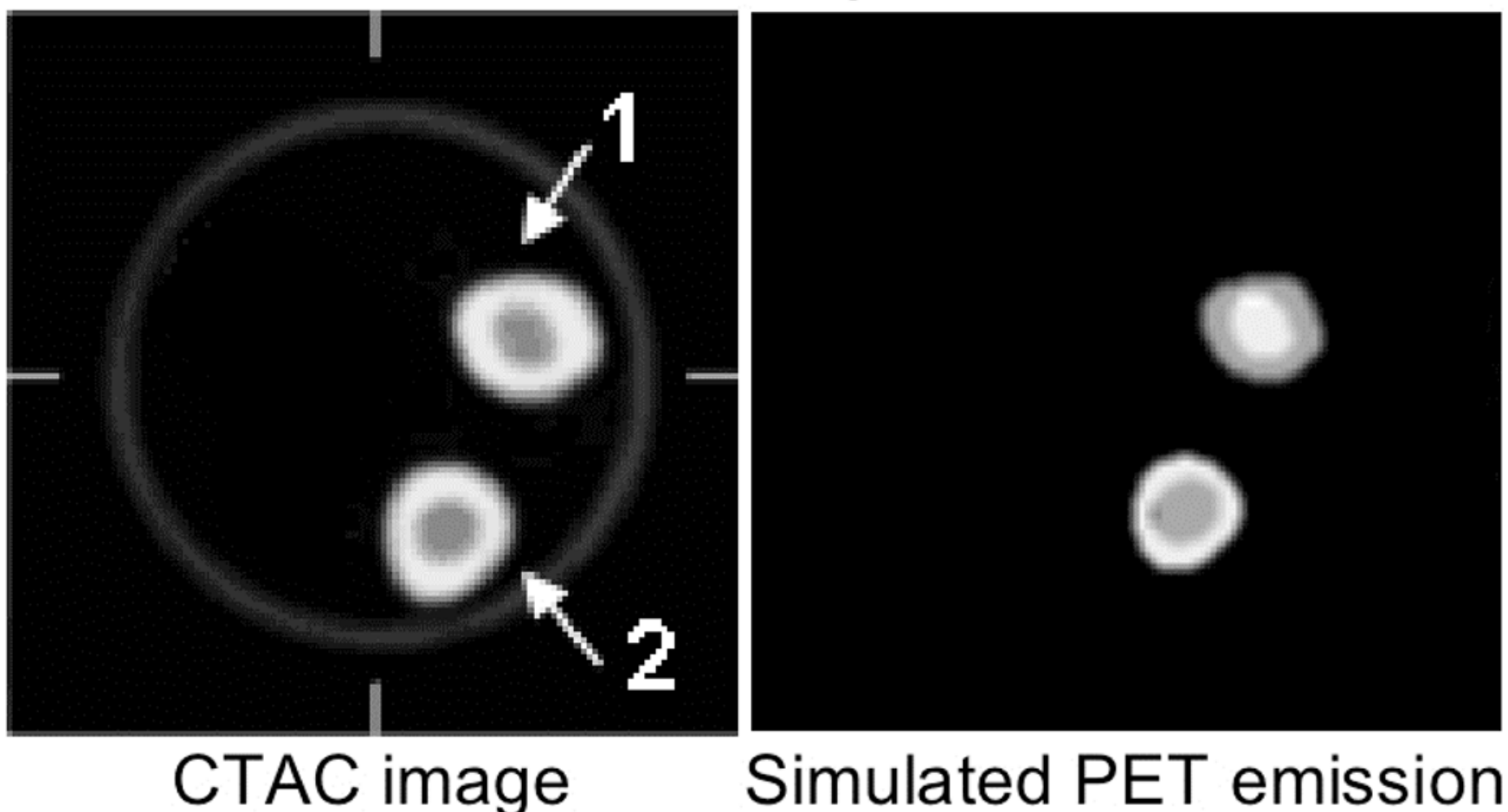

CTAC image

\section{Simulated PET emission}

Figure 4.

Transaxial sections of the CTAC and simulated PET emission images of tracer uptake in bone used to evaluate effect of CTAC errors on PET SUVs measured from bone tissue. 


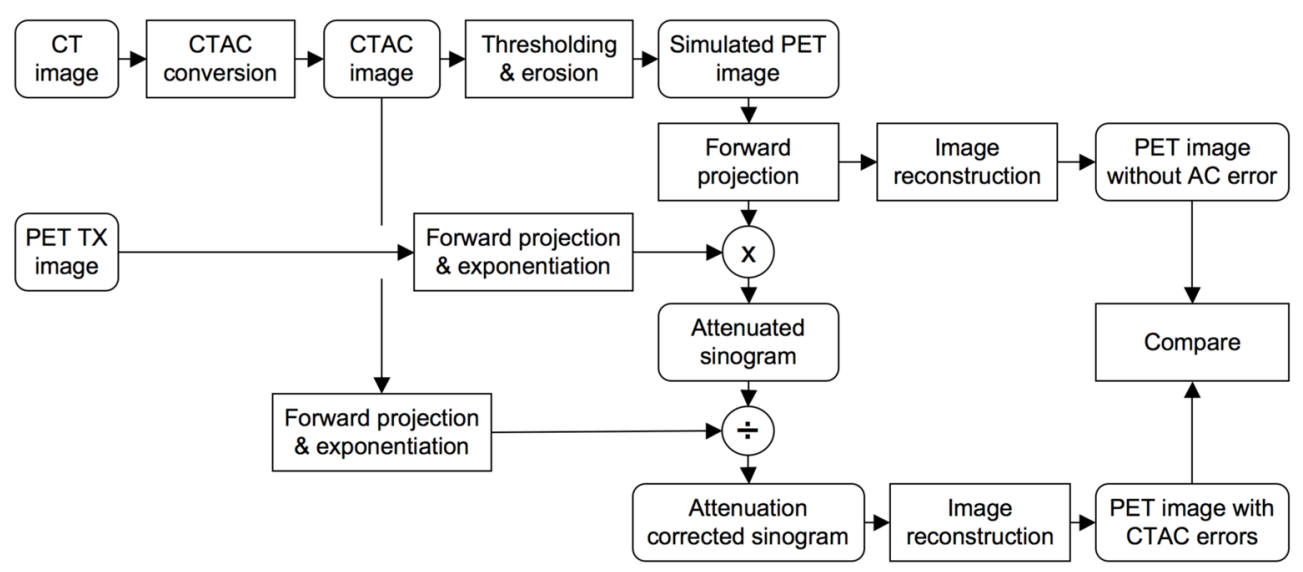

Figure 5.

Flowchart of the process followed to evaluate the bias in PET image quantification due to inaccuracies in the CTAC data using the reconstructed images of the bovine femur phantom. 


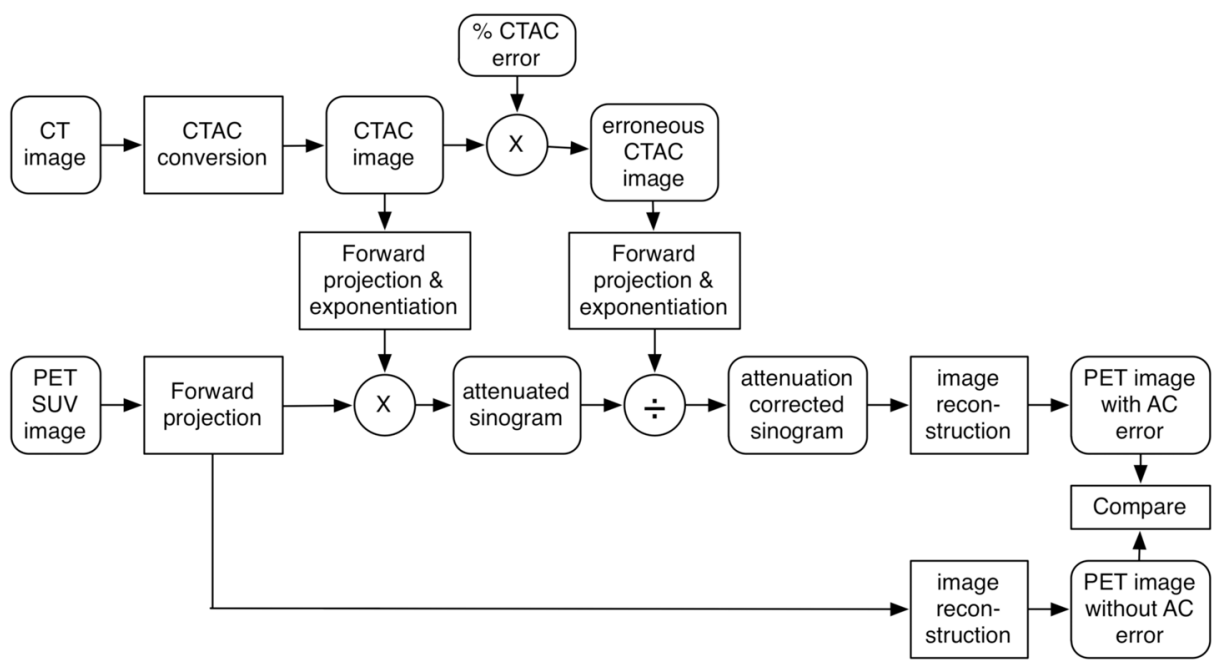

Figure 6.

Flowchart of the process followed to evaluate the bias in tumor quantification due to inaccuracies in CTAC data. 'CT image' and 'PET SUV image' are the reconstructed images for each patient. 

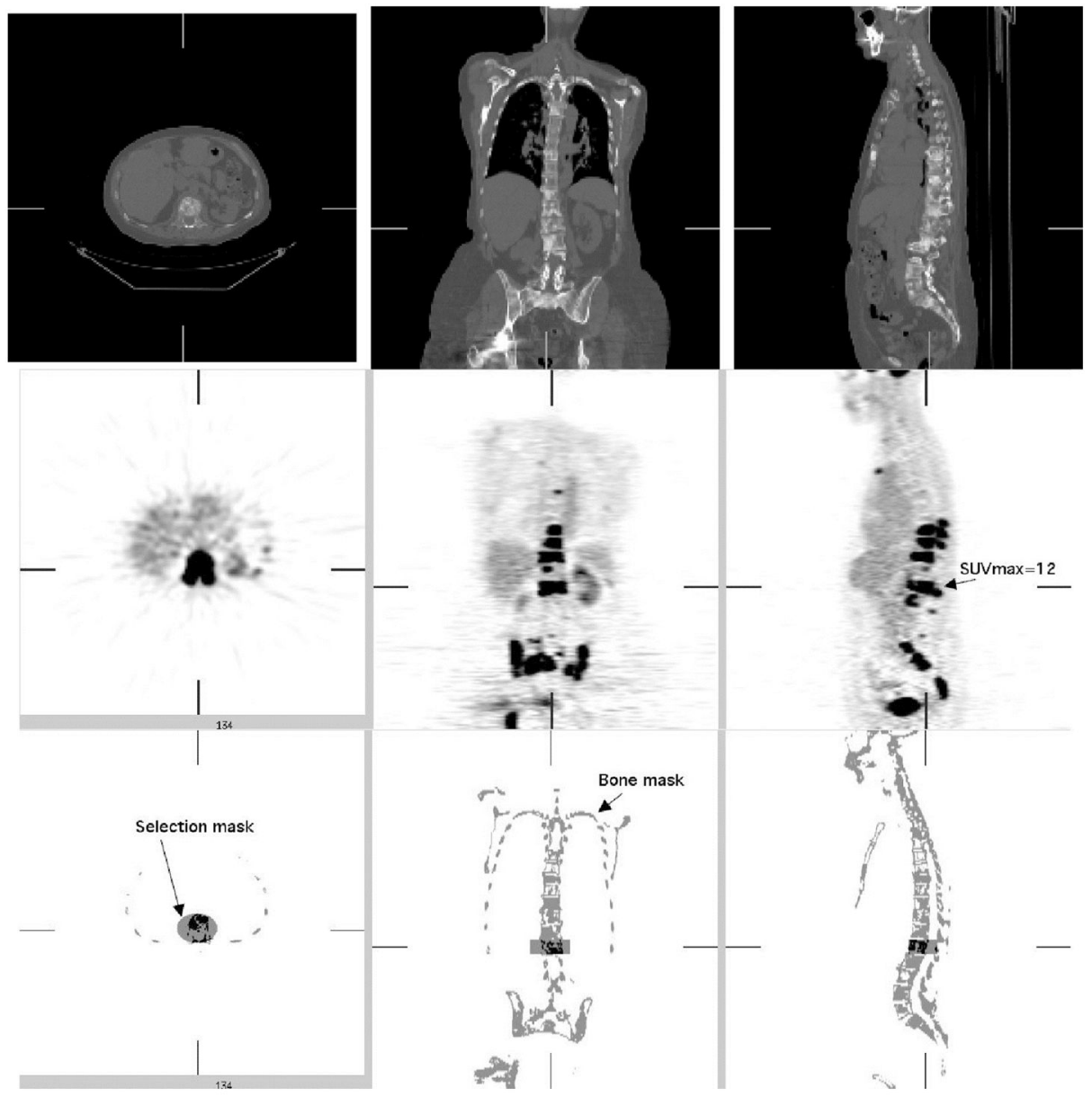

Figure 7.

Illustration of masking process used for one of the five PET/CT patient scans with significant FDG uptake in bone. Top: Axial, sagittal and coronal views of $70 \mathrm{~cm}$ diameter FOV CT image. Middle: PET FDG images. Bottom: Bone mask based on PET and CT images with one of the cylindrical selection masks used to isolate one vertebra for analysis. 

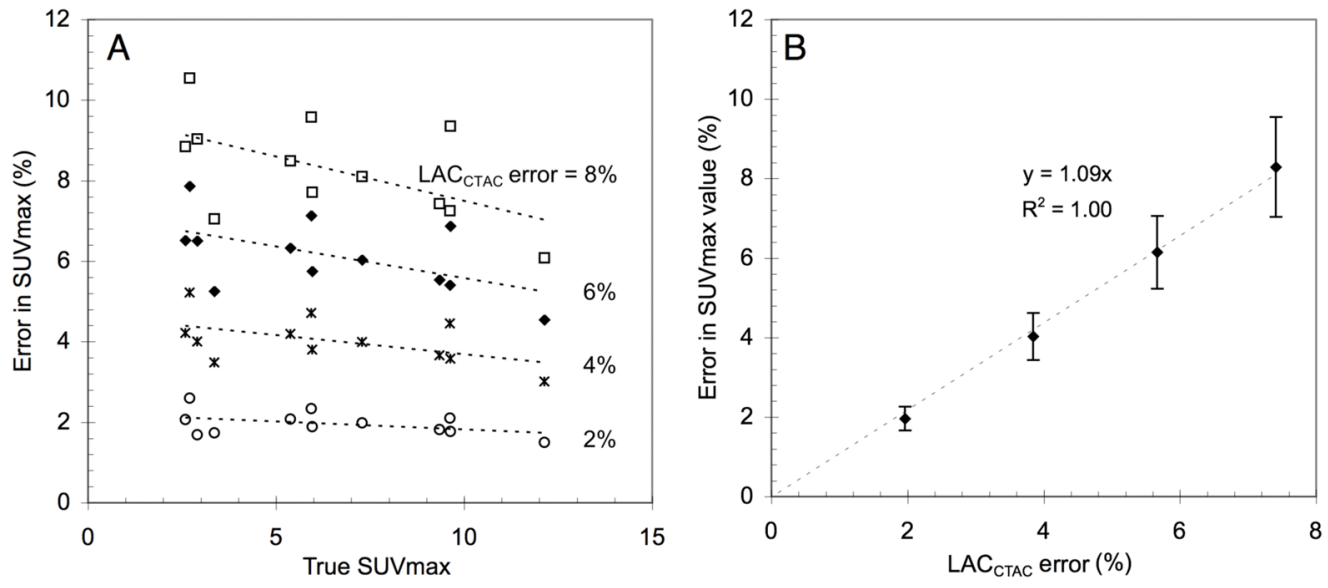

Figure 8.

A: SUV errors as a function of nominal SUV values for different values of percentage of CTAC error ( LAC $_{\mathrm{CTAC}}$ error). B: SUV errors as a function of percentage of error in CTAC $\left(\mathrm{LAC}_{\mathrm{CTAC}}\right.$ error (\%)) averaged over all nominal SUV values. 


\section{Table 2}

Assigned ('true') PET tracer uptake values in the bovine femur phantom for experiment 2.

\begin{tabular}{ll}
\hline Volume of Interest (VOI) & SUV \\
\hline Compact bone 1 & * \\
Compact bone 2 & 7.0 \\
Cancellous bone 1 & 7.0 \\
Cancellous bone 2 & 10.0 \\
\hline * Numbers correspond with those indicated in Figures 2 and 4.
\end{tabular}


$\stackrel{9}{9}$

일

ฮี

ڤ్

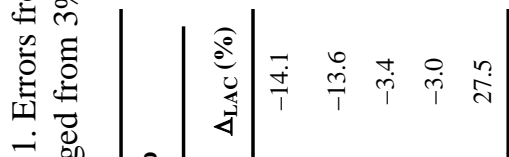




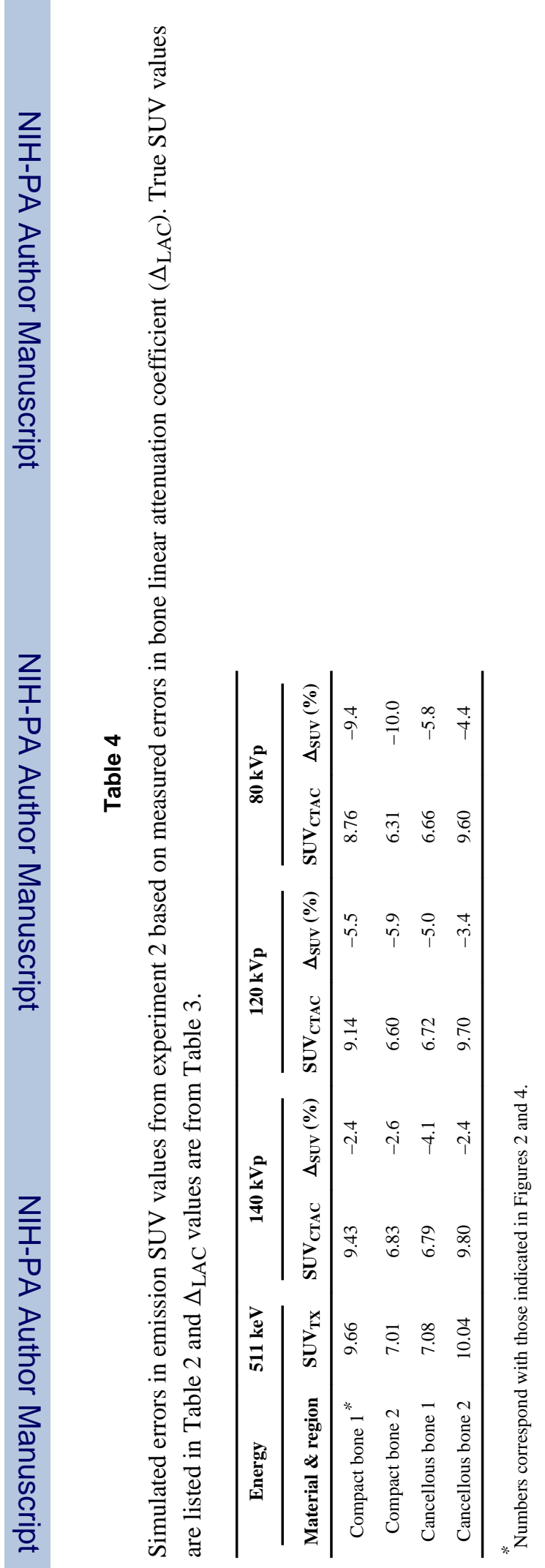

Phys Med Biol. Author manuscript; available in PMC 2013 May 07. 